“드 2013 IEEE. Personal use of this material is permitted. Permission from IEEE must be obtained for all other uses, in any current or future media, including reprinting/republishing this material for advertising or promotional purposes, creating new collective works, for resale or redistribution to servers or lists, or reuse of any copyrighted component of this work in other works." 


\title{
Using EEG Spatial Correlation, Cross Frequency Energy, and Wavelet Coefficients for the prediction of Freezing of Gait in Parkinson's disease patients
}

\author{
A.M. Ardi Handojoseno, James M. Shine, Tuan N. Nguyen, Member, IEEE, \\ Yvonne Tran, Simon J.G. Lewis, Hung T. Nguyen, Senior Member, IEEE
}

\begin{abstract}
Parkinson's Disease (PD) patients with Freezing of Gait (FOG) often experience sudden and unpredictable failure in their ability to start or continue walking, making it potentially a dangerous symptom. Emerging knowledge about brain connectivity is leading to new insights into the pathophysiology of FOG and has suggested that electroencephalogram (EEG) may offer a novel technique for understanding and predicting FOG. In this study we have integrated spatial, spectral, and temporal features of the EEG signals utilizing wavelet coefficients as our input for the Multilayer Perceptron Neural Network and k-Nearest Neighbor classifier. This approach allowed us to predict transition from walking to freezing with $87 \%$ sensitivity and $73 \%$ accuracy. This preliminary data affirms the functional breakdown between areas in the brain during FOG and suggests that EEG offers potential as a therapeutic strategy in advanced PD.
\end{abstract}

\section{INTRODUCTION}

Freezing of gait (FOG) is common in advanced Parkinson's Disease (PD) affecting over half of all patients after 5 years of illness [1]. In addition, up to a quarter of patients can experience FOG in the early stages of disease [2]. Patients with FOG are paroxysmaly unable to initiate or continue walking, feeling as if their feet are "glued" or "magnetized" to the ground. This symptom causes both physical and psychological distress and is a common cause of fall, often leading to social withdrawal with only partial amelioration from current treatments [1].

Several techniques have been explored to detect FOG such as using electromyogram (EMG) [3], acceleration sensors [4], functional neuroimaging [1] and electroencephalogram (EEG) [5]. Amongst these approaches, EEG offers predictive capability due to its greater temporal resolution and ability to measure dynamic physiological change. Previous work from our group using sub-band wavelet energy and total wavelet entropy of EEG signals has shown the ability of EEG to identify the onset of FOG before it appeared, with average

A.M. Ardi Handojoseno, Tuan N. Nguyen and Hung T. Nguyen are with Faculty of Engineering and Information Technology, University of Technology, Sydney, Broadway, NSW 2007, Australia. (AluysiusMariaArdi.Handojoseno@student.uts.edu, TuanNghia.Nguyen@uts.edu.au, Hung.Nguyen@uts.edu.au)

James M. Shine and Simon J.G. Lewis are with Parkinson's Disease Research Clinic, Brain and Mind Research Institute, University of Sydney, Level 4, Building F, 94 Mallet Street, Camperdown, NSW, 2050, Australia. (mac.shine@sydney.edu.au, simonl@med.usyd.edu.au)

Yvonne Tran is with the Key University Research Centre for Health Technologies, University of Technology, Sydney and the Rehabilitation Studies Unit, University of Sydney, Australia. (Yvonne.Tran@uts.edu.au) values of accuracy, sensitivity and specificity are $76 \%, 74$ $\%$, and $78 \%$, respectively [5].

A pathophysiological model of FOG has been proposed highlighting breakdown in the integration of information from across neural networks related to motor, cognitive and limbic functions in various regions of the brain [6]. This is aligned with recent research exploring the brain as a network of coupled dynamic systems with functional interactions between areas across the brain [7]. Several studies also give insight into Cortico-Subcortical cross-talk (CSC-ct) indicated by the coupling between slow wave (SW) and fast wave (FW) activity related to behavioural inhibition, motivation, emotion and decision making [8]. This paper attempts to establish a link between such bio-markers for the onset of FOG. We propose that based on wavelet decomposition and statistical analysis of its coefficients, both EEG sub-band cross spatial correlation (by means cross spectral correlation) and SW/FW EEG ratio can serve as electrophysiological signatures of FOG. We improved the performance of our work using this method with $87 \%$ classification sensitivity in predicting transition from walking to freezing.

\section{METHODS}

\section{A. Experimental Setup and Data Acquisition}

EEG signals were recorded in twenty-six PD patients with significant FOG (age $69.8 \pm 8.41$ ) during timed upand-go tasks (TUG) using EEG videomonitoring for FOG assessment at the Parkinson's Disease Research Clinic, the Brain and Mind Research Institute, University of Sydney. EEG was digitally recorded at 4 different locations on the scalp relating to their roles in the general control movement (O1-primary visual receiving area, P4-navigational movement area, Cz-primary motor area and Fz-supplementary motor area). The bipolar electrodes O1-T4 and P4-T3, and monopolar electrodes $\mathrm{Cz}$ and $\mathrm{Fz}$ with $\mathrm{CFz}$ as a reference were used in this study. All data were sampled at 500 Hz. Three groups of data were analyzed: normal walking, transition of FOG ( 5 seconds before freezing) and FOG.

\section{B. Data Preprocessing}

For this study, the first 10 patients without significant artifacts were selected, and 400 samples, each of 1 second length, were taken from each group (i.e. 1200 samples). Low frequency noise, high frequency noise and $50 \mathrm{~Hz}$ line frequency noise were eliminated using band-pass $(0.5-60 \mathrm{~Hz})$ 
and band-stop (50 Hz) Butterworth IIR filters. Stein's unbiased risk estimate thresholding based on wavelet transforms is used to remove ocular and muscular artifacts.

\section{Feature Extraction and Selection}

Wavelet transform (WT) is chosen to extract the individual EEG data due to its strengths in time-scale (frequency) localization and multirate filtering [9]. It is defined as the convolution between the signal $f(t)$ and the wavelet function $\psi_{u, s}(t)$ generated by dilations (contraction) and translation (shift) of mother wavelet $\psi(t)$

$$
W f(u, s)=f \star \bar{\psi}_{s}(u)=\int_{-\infty}^{+\infty} f(t) \frac{1}{\sqrt{s}} \psi^{*}\left(\frac{t-u}{s}\right)
$$

The discrete wavelet transforms (DWT) based on dyadic scales and position is used in which the EEG signal is decomposed into progressively finer details by means of multi-resolution analysis using complementary low and high pass filter, and is down sampled by 2 . The scaling coefficients as the result of low pass filter are further decomposed into low-pass and high-pass coefficients of WT. Each EEG sample $x(t)$ is then can be represented in terms of DWT as [9]

$$
x(t)=\sum_{k} c_{j_{0}}(k) \varphi_{j_{0}, k}(t)+\sum_{k} \sum_{j=j_{0}}^{\infty} d_{j}(k) \psi_{j, k}(t)
$$

where $\varphi_{j o, k}(t)$ and $\psi_{j, k}(t)$ denote the scaling function and wavelet expansion functions, respectively. The coefficients in this wavelet expansion, $c_{j_{0}}(k)$ and $d_{j}(k)$, under certain condition completely represent the original signal and can be used for description, analysis, approximation and filtering.

After six levels of decomposition, the coefficients retained from EEG samples at $500 \mathrm{~Hz}$ are $c_{6}(0-3.9 \mathrm{~Hz}), d_{6}(3.9-$ $7.8 \mathrm{~Hz}), d_{5}(7.8-15.6 \mathrm{~Hz}), d_{4}(15.6-31.3 \mathrm{~Hz})$, and $d_{3}(31.3-$ $62.5 \mathrm{~Hz})$. Noises $d_{2}(62.5-125 \mathrm{~Hz})$ and $d_{1}(125-250 \mathrm{~Hz})$ are discharged from further analysis as their magnitudes are negligible in a normal EEG. Daubechies wavelet of order 4 (db4) are used since its smoothing feature has been proved to work well in detecting changes of EEG signals [10]. Reconstruction of these five coefficients using the inverse wavelet transform approximately corresponding to the five physiological EEG sub-bands delta, theta, alpha, beta, and gamma, can be seen in Fig.1. The results of this filtering and decomposition are further processed to extract spatial, spectral and temporal parameters based on three feature extraction methods.

1) Wavelet Cross Spectrum : The brain can be seen as a complex network of interacting and coupled subsystems which determines its functions. Wavelet cross spectrum (WCS) based on the continues WT is used to investigate changes among EEG signals being recorded at different locations on the scalp. It can be interpreted as an indicator of functional relationship between different brain regions.

We calculate the Morlet wavelet transform of EEG subband electrodes at 4 different positions as a function of both time $t$ and frequency (scale) $s$, defined as [11]:

$$
W_{j}^{n}(t, s)=\frac{1}{\sqrt{s}} \sum_{\tau}^{T} x_{j}^{n}(\tau) \psi^{*}\left[\frac{(\tau-t)}{s}\right]
$$

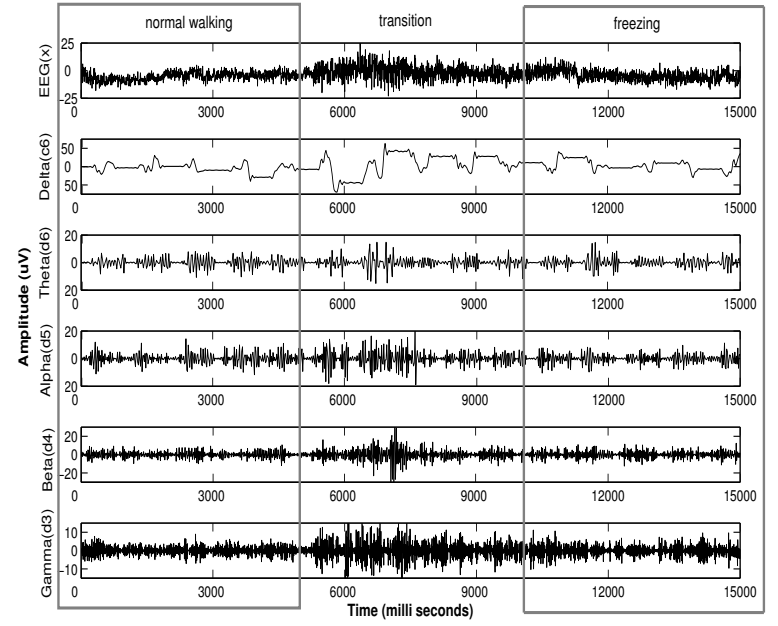

Fig. 1. Wavelet decomposition of EEG into five EEG sub-bands. It covers 3 conditions: normal walking, transition and freezing. Changing in wavelet energy between those three conditions are discernable.

where $\psi^{*}[]$ is the complex conjugate of the Morlet function. WCS measures the strength of a relationship between pairs of locations and can be defined as:

$$
W_{j k}^{n}(t, s)=W_{j}^{n}(t, s) W_{k}^{n}(t, s)^{*}
$$

The product of two spectra expressed by wavelet coefficients of the time scale representation of EEG sub-bands is a local measure of the interaction between signals $\mathrm{j}$ and $\mathrm{k}$ at the given frequency. Since Morlet function includes both real and imaginary part, as a result the WCS is complex valued. The average amplitude of this local wavelet cross correlation spectrum over time samples $t\left(\left|\overline{W C S_{j k}^{n}(t, s)}\right|\right)$ shows the global wavelet cross spectrum (GWCS) at related EEG sub-bands (see Fig.2). The first feature, total wavelet cross spectrum (TWCS) of EEG sub-bands, is obtained by calculating the sum of the global wavelet spectrum over scale (frequency) sub-bands. In addition, the centroid frequency wavelet cross spectrum (CFWCS) is calculated to show the center of gravity of each pair of signals at related EEG subbands and defined as follows [12]:

$$
C F=\frac{\sum_{i} f_{i} * P(f)}{\sum_{i} P(f)}
$$

where $f$ is a pseudo-frequency corresponding to the scale s, and $P(f)$ is the GWCS power.

2) Wavelet Cross Frequency Energy Ratios: The coupling between slow and fast frequency in the EEG spectrum has been studied as an indicator of cortico-subcortical cross-talk [8]. Studying the relations between the different frequency bandwidth could reveal some of the physiological dynamics of brain function related to different arousal systems. In PD research using quantitative EEG, some EEG electrode locations showed increased SW (delta and theta) and decreased FW (alpha and beta) which correlate with cognition decline in PD patients [13].

Wavelet energy is computed on each retained wavelet 


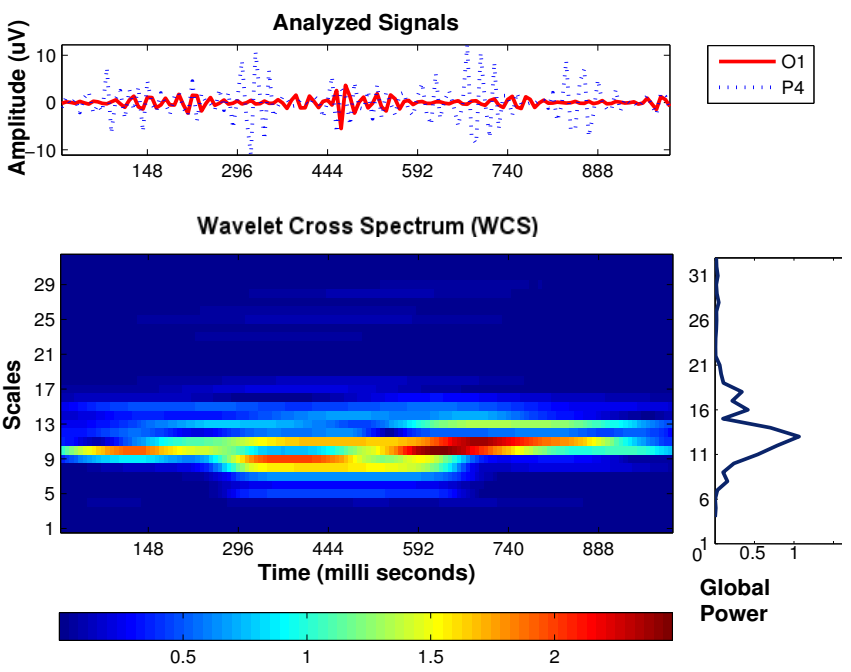

Fig. 2. Wavelet cross spectrum for transition of freezing at gamma subband. The upper panels shows EEG signals from electrode pairs $\mathrm{O} 1$ and P4. The right panel presents the global wavelet spectrum obtained by averaging over time samples.

scale $\left(c_{6}, d_{6}, d_{5}, d_{4}, d_{3}\right)$ by squaring and summing the wavelet coefficients of the decomposed level [5]. In this study, wavelet cross frequency energy ratios $(\mathrm{ER})(\boldsymbol{\delta} / \boldsymbol{\beta})$ and $(\theta / \beta)$ were observed and selected as features since they are part of major frequency bands of oscillation in the basal ganglia which may have a functional role in movement [14].

3) Statistical Features: Various simple statistical properties of the EEG time series have already been used in preceding brain studies. It was proven to be effective for discriminating different brain conditions. In this study some popular methods were applied to extract information from wavelet coefficients of the EEG epoch $x(t)$ : quantifying the central tendency (mean), degree of dispersion (standard deviation), asymmetry (skewness) and peakedness (kurtosis, maximum, minimum). They were used to represent the time frequency distribution of the EEG signals in each sub-band.

In view of the data having a non-normal distribution and to reduce false positives arising from the large dataset, nonparametric statistical analysis Wilcoxon sum rank test with continuity correction of 0.5 was implemented. Only features with significant statistical differences between those groups of data ( $p$-value $<0.05$ ) were chosen for further processing.

\section{Classification}

Multilayer perceptron neural network (MLP) analysis was used to classify data samples based on selected features. The Levernberg Marquardt algorithm was chosen as a training method of a three layer MLP with $56 \%, 25 \%$ and $19 \%$ of the data used for training, validation and testing, respectively. The validation set was used as a stopping criterion to avoid overfitting as well as error goal 0.01 in single MLP with 8 to 12 hidden layer neurons. For comparison, the obtained features were also used to train and test using k-nearest neighbors (kNN) classifier with 15 to 40 nearest neighbors based on the Euclidian distance. Each feature and their
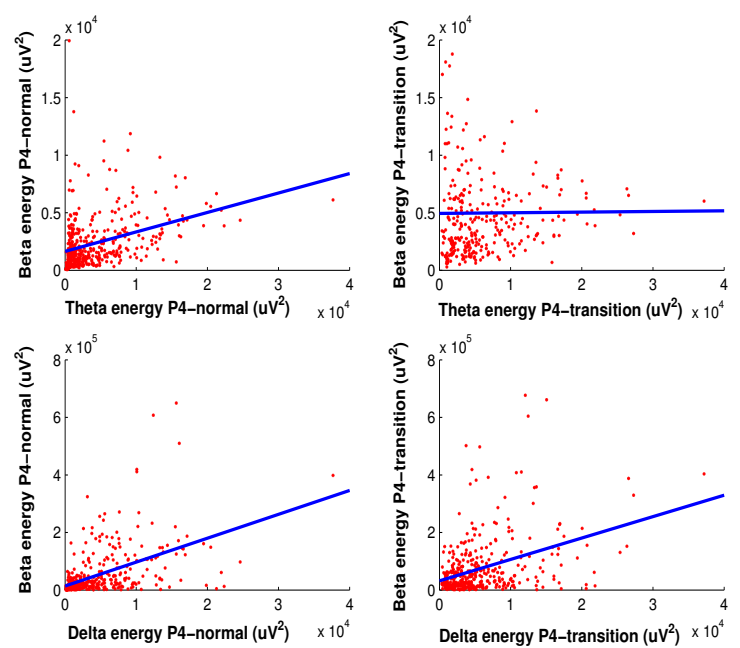

Fig. 3. Scatterplots of significant $\theta / \beta$ (electrode $\mathrm{P} 4$ ) correlation in the normal walking condition which is different with no significant correlation in the transition five seconds before freezing condition (upper panel). Two bottom scatterplots show a non-significant difference between $\delta / \beta$ (electrode P4) correlation in normal walking condition and the transition five seconds before freezing condition.

combination were trained and tested for twenty times and the mean result was recorded.

\section{RESULTS AND DISCUSSION}

The selected spatial and spectral features with $p$-value $<0.05$ are presented in Table 1 from two of four features (CFWCS and ER). One can see that the freezing condition in PD patients significantly affected neural synchronization of different brain regions. Amongst four different regions under study, $\mathrm{P} 4$ and $\mathrm{Cz}$ appeared as the most important sites contributing in nine out of sixteen most important CFWCS features selected for their statistical significance during the transition of freezing (N-T). This result supports our view in the previous report that the freezing condition has a deep impact in the area that is responsible for integrating sensory information from various parts of the body [5].

The assessment of WCS between each EEG channel pair and CFWCS in each channel demonstrated an altered pattern of synchronisation in the theta sub-band during transition from walking to freezing and and in the freezing period. This finding is aligned with prior studies in FOG and PD in general, which suggests this activity is associated with motor-task changing [15]. In addition, since the freezing phenomenon has also been associated with non-motor features, the coupling of delta and beta oscillation during the transition and freezing periods may represent factors such as increased cognitive activity, a rise in anxiety levels or other perceptual processing [16]. Fig. 3 show the dynamics of subband wavelet energy as a result of transition from walking to freezing in electrodes $\mathrm{P} 4$ which have significant changing in $\theta / \beta$ but not in $\delta / \beta$.

For classification, only features that were statistically significant ( $p$-value $<0.05$ ) between two conditions were taken for input. We took 131 features representing the spatial spectral temporal characteristics of EEG signals based on spectral 
TABLE I

Sensitivity of Centroid Frequency Wavelet Cross Spectrum AND WAVELET CRosS FREQUENCY ENERGY RATIOS FEATURES

\begin{tabular}{cccccc}
\hline Feature & $\begin{array}{c}\mathrm{N}-\mathrm{T} \\
p \text {-value }\end{array}$ & $\begin{array}{c}\mathrm{N}-\mathrm{F} \\
p \text {-value }\end{array}$ & Feature & $\begin{array}{c}\mathrm{N}-\mathrm{T} \\
p \text {-value }\end{array}$ & $\begin{array}{c}\mathrm{N}-\mathrm{F} \\
p \text {-value }\end{array}$ \\
\hline CF-O1P4- $\beta$ & 0.0047 & 0.0659 & CF-P4Cz- $\alpha$ & 0.0005 & 0.1258 \\
CF-O1Cz- $\gamma$ & 0.0001 & 0.0257 & CF-P4Cz- $\theta$ & 0.0001 & 0.2431 \\
CF-O1Cz- $\beta$ & 0.0003 & 0.0037 & CF-P4Fz- $\gamma$ & $\leq 0.0001$ & $\leq 0.0001$ \\
CF-O1Cz- $\alpha$ & 0.0001 & 0.0032 & CF-P4Fz- $\beta$ & 0.0012 & $\leq 0.0001$ \\
CF-O1Cz- $\theta$ & 0.0139 & 0.4326 & CF-P4Fz- $\alpha$ & 0.0078 & $\leq 0.0001$ \\
CF-O1Fz- $\theta$ & 0.0001 & 0.0001 & CF-P4Fz- $\theta$ & 0.0060 & $\leq 0.0001$ \\
CF-P4Cz- $\gamma$ & $\leq 0.0001$ & 0.0121 & CF-CzFz- $\gamma$ & 0.0004 & 0.7581 \\
CF-P4Cz- $\beta$ & 0.0031 & 0.0250 & CF-CzFz- $\theta$ & 0.0001 & 0.0001 \\
\hline$\delta / \beta$ O1 & 0.0079 & 0.5862 & $\theta / \beta$ O1 & $\leq 0.0001$ & 0.0431 \\
$\delta / \beta$ Cz & $\leq 0.0001$ & 0.0288 & $\theta / \beta$ P4 & $\leq 0.0001$ & 0.2335 \\
$\delta / \beta$ Fz & $\leq 0.0001$ & $\leq 0.0001$ & $\theta / \beta$ Cz & $\leq 0.0001$ & $\leq 0.0001$ \\
\hline N: normal walking; T: transient; F: freezing
\end{tabular}

cross correlation (magnitude and centroid frequency), cross frequency energy ratios and statistics of wavelet energies of EEG signals from 3 different conditions. The discriminant capability of each feature and their combination in differentiating normal walking and transition to freezing were then examined.

The performance of classification did not necessarily increase when different features were combined. As can be seen in Table 2, utilizing MLP, the highest result for sensitivity has been achieved using only WCS (83.37\% mean) whilst applying only the statistic features as the input of classifiers gave the best result for accuracy (73.47\% mean). Interestingly, WCS gave the best result for accuracy in differentiating normal walking and transition to freezing using $\mathrm{kNN}$ (72\% mean) while the combination of all features has increased sensitivity up to $87.25 \%$. However, both MLP and kNN consistently showed the strength of WCS in correctly detecting a transition condition, which presumably related to the combined information within this metric (spatial, spectral, and temporal). While stronger ability to correctly assess both conditions (accuracy) is still on the agenda, how good the test is at detecting freezing is determined by its sensitivity, as we are more concerned on correctly predicting transient/freezing than correctly predict normal walking. Our result suggest that -except for CFWCSall other features (WCS, ER, and statistical information of wavelet coefficients) represent good candidates for further development.

TABLE II

CLASSIFICATION RESULTS OF PROPOSED FEATURES USING MLP-NN AND KNN CLASSIFIER IN DETECTING TRANSITION 5 SECOND BEFORE FREEZING FROM NORMAL WALKING

\begin{tabular}{|c|c|c|c|c|c|c|}
\hline \multirow{2}{*}{$\begin{array}{c}\text { Inputs } \\
\text { Features }\end{array}$} & \multicolumn{3}{|c|}{ MLP } & \multicolumn{3}{|c|}{ kNN } \\
\hline & Se $\%$ & Sp \% & Acc \% & Se $\%$ & Sp \% & Acc $\%$ \\
\hline WCS & 83.37 & 57.63 & 69.9 & 83.50 & 60.50 & 72.00 \\
\hline CFWCS & 63.51 & 62.6 & 62.73 & 64.25 & 61.50 & 62.87 \\
\hline ER & 77.60 & 59.02 & 68.13 & 61.00 & 54.75 & 57.88 \\
\hline Stat & 75.47 & 71.47 & 73.47 & 82.75 & 57.25 & 70.00 \\
\hline WCS,Stat & 79.19 & 66.57 & 73.00 & 83.75 & 59.00 & 71.38 \\
\hline WCS,Stat,ER & 74.28 & 69.89 & 72.18 & 87.00 & 52.00 & 69.50 \\
\hline All & 76.27 & 67.29 & 71.75 & 87.25 & 52.75 & 70.00 \\
\hline
\end{tabular}

\section{CONCLUSIONS AND FUTURE WORK}

This study demonstrates that a novel approach combining the spatial, spectral and temporal features of surface EEG recording may prove effective for the prediction of FOG in PD. In addition, a greater understanding of these recordings will offer insight into the pathophysiological mechanisms underlying this devastating symptom. We propose that this higher sensitivity for event prediction has been achieved due to the integration of features measuring a dynamic pattern of space-time-frequency in EEG signals during transition to a freezing condition. The application of all four features proposed in this study has increased capacity to correctly detect a FOG attack. Therefore, they can serve as useful parameters in discriminating EEG signals. Future work will hone the use of these electrophysiological parameters, with dimensional reduction and classification methods that should hopefully improve the utility of this approach.

\section{REFERENCES}

[1] Y. Okuma, Freezing on gait in Parkinson's disease, J. Neurol, no. 253, Dec. 2006, pp. 27-32.

[2] S.T. Moore, H.G. MacDougall, and W.G. Ondo, Ambulatory monitoring of freezing of gait in Parkinson's disease, J Neuroscience Meth, vol. 167 , no. 2, pp. 340-348, 2008.

[3] M.B. Popovic, M.D. Jovicic, S. Radovanovic, I. Petrovic, and V. Kostic, A simple method to assess freezing of gait in PD patients, Braz J Med Biol Res, vol. 43, no. 9, pp. 883-889, Sep. 2010.

[4] S. Mazilu, M. Hardegger, Z. Zhu, D. Roggen, G. Troster, M. Plotnik, and J.M. Hausdorff, Online detection of freezing of gait with smartphones and machine learning techniques, in Proc. 6th Int. Conf. Pervasive Computing Technologies for Healthcare, 2012, pp. 123-130.

[5] A.M. Handojoseno, J.M. Shine, T.N. Nguyen, Y. Tran, S.J.G. Lewis, and H.T. Nguyen, The detection of Freezing of Gait in Parkinson's disease patients using EEG signals based on Wavelet decomposition, in Proc. 34th Annu. Int. Conf. IEEE EMBC, 2012, pp. 69-72.

[6] S.J.G. Lewis and R. A. Barker, A pathophysiological model of freezing of gait in PD,in Park. Relat. Disord. vol. 15, no.5, pp. 333-338, 2009.

[7] C.J. Stam, Nonlinear dynamical analysis of EEG and MEG, in Clinical Neurophysiology, vol. 116, no. 10, pp. 2266-2301, 2005

[8] D.G.L. Schutter, C. Leitner, J.L. Kenemans, and J. van Honk, Electrophysiological correlates of cortico-subcortical interaction: A crossfrequency spectral EEG analysis, in Clinical Neurophysiology, vol. 117, no. 2, pp. 381-387, 2006.

[9] Burrus, C. Sidney, et al, Introduction to wavelets and wavelet transforms: a primer, Vol. 23, Upper Saddle River: Prentice hall, 1998.

[10] A. Subasi, EEG signal classification using wavelet feature extraction and a mixture of expert model, in Expert Systems with Applications, vol.32 no.4, pp. 1084-1093, 2007.

[11] D. Lee, Analysis of phase-locked oscillations in multi-channel singleunit spike activity with wavelet cross-spectrum, J Neuroscience Meth., vol. 115 , no.1, pp. 67-75, 2002.

[12] L.B. Nguyen, A. V. Nguyen, S. H. Ling, and H. T. Nguyen, A PSObased neural network for detecting nocturnal hypoglycemia using EEG signals, in Proc. 2012 Int. Joint Conf. Neural Networks, 2012, pp. 1-6.

[13] J.N. Caviness, J.G. Hentz, V.G. Evidente, E. Driver-Dunckley, J. Samanta, P. Mahant, and C.H. Adler, Both early and late cognitive dysfunction affects the electroencephalogram in Parkinson's disease, in Parkinsonism Relat. Disord., vol. 13, no.6, pp. 348-354, 2007.

[14] P. Brown, Oscillatory nature of human basal ganglia activity: relationship to the pathophysiology of Parkinson's disease, in Mov. Disord. vol. 184, no. 4, pp 357-363, 2002.

[15] S.J. Palmer, P.W.H. Lee, Z.J. Wang, W.L. Au, and M.J. Mc Keown, $\theta, \beta$ but not $\alpha$-band EEG connectivity has implications for dual task performance in Parkinsons disease, in Parkinsonism Relat. Disord, vol 16, 2010, pp. 393-397.

[16] G.G. Knyazev, J.L.G. Dennis, and J. van Honk, Anxious apprehension increases coupling of delta and beta oscillations, Int. J. Psychophysiology, vol. 61, no. 2, pp. 283-287, 2006. 